\title{
Actividad de AIA oxidasa en estacas de especies del género Nothofagus
}

\author{
IAA oxidase activity on species of Nothofagus genus cuttings
}

\author{
Mirtha Latsague ${ }^{a *}$, Patricia Sáez ${ }^{a}$, Marco Paredes ${ }^{a}$, Edith Alarcón ${ }^{b}$

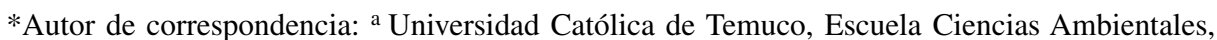 \\ Facultad de Recursos Naturales, casilla 15-D, Temuco, Chile, Tel.: 56-45-205409, mlatsagu@uct.cl \\ b Universidad Católica de Temuco, Escuela de Ciencias Forestales, Facultad de Recursos Naturales, Temuco, Chile.
}

\begin{abstract}
SUMMARY
There is variation in rooting capacity among the different species of Nothofagus. Lenga cuttings (N. pumilio) present an almost null answer to rooting, compared to raulí (N. alpina) and roble ( $N$. obliqua). According to antecedents compiled in literature, the endogenous levels of indole acetic acid (IAA), hormone responsible for the formation of adventitious roots, vary depending on the levels of IAA oxidase activity. Based on these observations, the IAA oxidase activity was comparativily analyzed in cuttings from these three Nothofagus species. To quantify the enzymatic activity, a spectrophotometric test was carried out, measuring at $535 \mathrm{~nm}$. The residual amount of IAA was determined by reference to a calibration curve. The enzymatic activity was expressed in IAA $\mathrm{mM} \mathrm{mg}$ protein $^{-1}$ hours $^{-1}$. The results show that lenga presents a greater IAA oxidase enzymatic activity when compared to the other analyzed species $(P<0.05)$. These results would support at least partially the differences in the rootting capacity in Nothofagus species studys.
\end{abstract}

Key words: Nothofagus, rooting, AIA oxidasa, indole acetic acid.

\section{RESUMEN}

Entre las distintas especies de Nothofagus existe variación en la capacidad de enraizamiento. Las estacas de lenga (N. pumilio) presentan una respuesta casi nula al enraizamiento, comparada con raulí ( $N$. alpina) y roble ( $N$. obliqua). Según antecedentes recopilados en literatura, los niveles endógenos de ácido indol acético (AIA), hormona responsable de la formación de raíces adventicias, varían según los niveles de actividad de AIA oxidasa (AIA-O). Basados en estas observaciones, la actividad de AIA-O fue comparativamente analizada en estacas de estas tres especies de Nothofagus. Para cuantificar la actividad enzimática se realizó un ensayo de espectrofotometría, midiendo a $535 \mathrm{~nm} \lambda$. La cantidad residual de AIA se estimó por referencia a una curva de calibración. La actividad enzimática fue expresada en $\mathrm{mM}$ de AIA mg de proteína ${ }^{-1} \mathrm{~h}^{-1}$. Los resultados muestran que $N$. pumilio presenta una mayor actividad enzimática de AIA oxidasa en comparación con las otras especies analizadas $(P<0,05)$. Estos resultados respaldarían, al menos parcialmente, las diferencias en la capacidad de rizogénesis en las especies de Nothofagus estudiadas.

Palabras clave: Nothofagus, enraizamiento, AIA oxidasa, ácido indol acético.

\section{INTRODUCCIÓN}

La presión del hombre sobre los bosques nativos para obtener productos tangibles promueve la desaparición y degradación de ecosistemas. Por esta razón el manejo forestal se debe llevar a cabo desde una perspectiva de sustentabilidad ecológica y para ello la silvicultura debe ser compatible con las estrategias de regeneración de las plantas (Dezzotti et al. 2003). Es aquí donde la propagación vegetativa y en particular el arraigamiento de estacas puede usarse como una herramienta para producir material genético de alto valor que podría emplearse para el enriquecimiento de bosques (Santelices y Cabello 2006).
La amplia distribución de las especies chilenas del género Nothofagus, así como la gran diversidad de hábitat en que se desarrollan, permite que se manifieste variabilidad en las características ecofisiológicas de las especies (Veblen y Donoso 1987). Esta variabilidad se ve reflejada en la capacidad que muestran algunas especies del género de reproducirse vegetativamente a través de rebrote de tocón.

En el arraigamiento de las especies más comunes del género Nothofagus (N. obliqua (Mirb.) Oerst., $N$. alpina (Poepp. et Endl.) Oerst. y N. pumilio (Poepp. et Endl.) Krasser) se han detectado diferencias respecto a su capacidad de formar raíces adventicias (Silva 1968, 
Santelices 1993, Latsague y Lara 2003). Estas diferencias se atribuyen, entre otros factores, a la actividad de la enzima AIA oxidasa (AIA-O), responsable de los niveles endógenos de la auxina ácido indol acético, regulador de crecimiento que participa del proceso de rizogénesis. Existe controversia respecto a la relación entre actividad de AIA-O y el proceso de rizogénesis. Günes (2000) observó una correlación positiva entre el enraizamiento y la actividad de AIA-O en especies de Populus. Por otro lado, Qaddoury y Amssa (2003) informan que la formación de raíces en estacas de palma (Phoenix dactylifera $\mathrm{L}$.) se caracteriza por una clara disminución en la actividad de AIA-O al inicio del proceso de rizogénesis y un marcado aumento posteriormente. Liu et al. (1996) señalan que el contenido endógeno de AIA aumenta con la disminución de la actividad de AIA-O durante la formación de raíces adventicias en hipocótilos de soya (Glycine max Merr).

Basados en los antecedentes señalados, el objetivo de esta investigación fue determinar y comparar la actividad de la enzima AIA-O en estacas de tres especies del género Nothofagus (N. obliqua, N. alpina y N. pumilio) con distintas capacidades de respuesta al enraizamiento.

\section{MÉTODOS}

Extracción y purificación parcial de proteínas. Las estacas de cada una de las especies de Nothofagus en estudio fueron colectadas de plantas adquiridas en el vivero perteneciente a la Corporación Nacional Forestal (CONAF), Curacautín, Provincia de Malleco, IX Región, y congeladas en nitrógeno líquido hasta el momento de su análisis. De estas estacas se pesó $1,5 \mathrm{~g}$, los que se molieron en nitrógeno líquido hasta obtener un polvo fino que se homogenizó en $7 \mathrm{~mL}$ de tampón de extracción (fosfato de potasio $50 \mathrm{mM}, \mathrm{pH}$ 6,6). El homogeneizado se centrifugó dos veces a 3.500 rpm durante 15 minutos a $4{ }^{\circ} \mathrm{C}$ y cada vez se desechó el precipitado. El segundo sobrenadante constituyó el extracto crudo. A $10 \mathrm{~mL}$ de extracto crudo se le agregaron tres volúmenes de $30 \mathrm{~mL}$ de acetona $50 \%$ v/v y se dejó reposando a $-20{ }^{\circ} \mathrm{C}$ durante $4 \mathrm{~h}$. Después de este tiempo la mezcla se centrifugó a $3.500 \mathrm{rpm}$ durante 40 minutos a $4{ }^{\circ} \mathrm{C}$. El precipitado obtenido se disolvió en $4 \mathrm{~mL}$ de tampón fosfato de potasio $50 \mathrm{mM} \mathrm{pH} \mathrm{6,6,} \mathrm{obteniendo} \mathrm{de}$ esta forma la fracción proteica. Se determinó la concentración de proteínas por el método de Bradford (1976) usando seroalbúmina de bovino como estándar proteico.

Ensayo de actividad enzimática. Para este ensayo se utilizó una concentración estándar de proteína correspondiente a $30 \mu \mathrm{g} \mathrm{mL}^{-1}$. La actividad de la enzima AIA-O fue medida con el método espectrofotométrico descrito por Beffa et al. (1990). La mezcla de la reacción consistió en: 0,76 $\mathrm{mL}$ de tampón fosfato de potasio $50 \mathrm{mM}$ a $\mathrm{pH} 6,0 \mathrm{con}$ $0,01 \mathrm{~mL}$ de cloruro de manganeso $5 \mathrm{mM} ; 0,01 \mathrm{~mL}$ de 2-4-diclorofenol $5 \mathrm{mM}, 0,02 \mathrm{~mL}$ de AIA $1,4 \mathrm{mM}$, y 0,2 $\mathrm{mL}$ de extracto proteico. El volumen final de la mezcla de la reacción fue de $1,0 \mathrm{~mL}$. El ensayo se mantuvo por 1 hora a $25{ }^{\circ} \mathrm{C}$ en oscuridad. Para determinar actividad enzimática AIA-O se utilizó el reactivo de Salkowski, midiendo absorbancia a longitud de onda de $535 \mathrm{~nm}$. Las absorbancias obtenidas fueron referidas a la ecuación de la curva de calibración con AIA según Latsague y Sáez (2006), obteniéndose las correspondientes concentraciones de AIA remanente. Una unidad de actividad AIA-O es equivalente a $1 \mathrm{mM}$ AIA mg proteína ${ }^{-1} \mathrm{~h}^{-1}$.

Diseño experimental y análisis estadístico. Se diseñaron tres bloques al azar con cinco repeticiones cada uno (cada bloque representó una especie). Para validar los resultados de actividad enzimática y determinar diferencias estadísticamente significativas entre las especies se aplicó un análisis de varianza a través de un ANDEVA simple y la prueba $t$ de Student $(P<0,05)$.

\section{RESULTADOS}

Determinación de proteínas y actividad enzimática en los extractos. El valor promedio $( \pm S)$ de proteínas medido en los extractos crudos de cada una de las especies fue de $47 \pm 10 \mu \mathrm{g} \mathrm{mL}^{-1}$ para $N$. pumilio, $67 \pm 8 \mu \mathrm{g} \mathrm{mL}^{-1}$ para $N$. alpina, y $60 \pm 6 \mu \mathrm{g} \mathrm{mL}-1$ para $N$. obliqua, sin encontrar diferencias significativas entre las especies $(P>0,05)$. Los valores parciales de concentración de proteínas solubles totales en las estacas de las especies de Nothofagus estudiadas se encontraron en un rango de 30 a $80 \mu \mathrm{g} \mathrm{mL}^{-1}$, lo que hizo necesario estandarizar la concentración inicial de proteínas a $30 \mu \mathrm{g} \mathrm{mL}^{-1}$ en cada una de las réplicas y sobre esta concentración se determinó actividad enzimática.

Se observó mayor actividad de AIA-O en el tejido de estacas de $N$. pumilio $(1,389 \pm 0,0018 \mathrm{mM}$ AIA mg proteína $\left.{ }^{-1} \mathrm{~h}^{-1}\right)$ comparado con extractos de estacas de $N$. alpina $\left(1,381 \pm 0,0048 \mathrm{mM}\right.$ AIA mg proteína $\left.{ }^{-1} \mathrm{~h}^{-1}\right)$ y $N$. obliqua $\left(1,379 \pm 0,0038 \mathrm{mM}\right.$ AIA mg proteína $\left.{ }^{-1} \mathrm{~h}^{-1}\right)$. Se encontraron diferencias significativas en los valores promedio de actividad enzimática de $N$. pumilio comparada con $N$. alpina y $N$. obliqua $(P<0,05)$.

\section{DISCUSIÓN Y CONCLUSIONES}

Los resultados muestran variaciones de la actividad enzimática en tejidos de estacas de diferentes especies de Nothofagus. Así, para N. pumilio, especie con dificultad en la diferenciación de raíces adventicias, hay una mayor actividad de la enzima AIA-O. Por otro lado, la menor actividad enzimática encontrada en $N$. obliqua explicaría la mayor capacidad rizogénica informada por Santelices (1993). 
La formación de raíces adventicias depende de una compleja interacción entre niveles endógenos de AIA y actividad AIA-O. Es así como la rizogénesis en tallos de castaño (Castanea sativa Mill.) cultivados in vitro está acompañada por una disminución de la actividad AIA-O en la parte basal de la estaca y, como consecuencia de ello, podrían aumentar los niveles de AIA en la región de enraizamiento del tallo (Mato y Vieitez 1986). Por el contrario, en las estacas no enraizadas, la actividad AIA-O permanece alta. La mayor actividad enzimática podría influir en la disminución de los niveles endógenos de AIA libre. De acuerdo a lo descrito por Qaddoury y Amssa (2003), periodos de alta actividad enzimática probablemente se correlacionan con una reducción de los niveles endógenos de auxina. Estos mismos autores informan que durante la formación de raíces adventicias en palma (Phoenix dactylifera) se observa disminución en la actividad de AIA-O.

Los resultados podrían indicar que la alta actividad enzimática detectada en $N$. pumilio podría interferir en la formación de raíces adventicias, debido a una posible disminución de los niveles endógenos de auxinas.

Considerando que la técnica de enraizamiento de estacas contribuye a fomentar el manejo sustentable y la protección de los bosques nativos, los resultados presentados aportan al conocimiento de las características fisiológicas de estas especies y su relación con la regeneración de plantas, sobre todo si se piensa realizar algún tipo de mejoramiento utilizando la técnica de estaquillado, para contribuir en programas de reforestación y recuperación de bosque nativo.

\section{REFERENCIAS}

Beffa R, M Hilary, P Pilet. 1990. In vitro oxidation of indoleacetic acid by soluble auxin - oxidases and peroxidases from maize roots. Plant Physiology 94: 485-491.

Bradford M. 1976. A rapid sensitive method for the quantitiation of microgram quantities of protein utilizing the principle of protein dye - binding. Analytical Biochemistry 72: 248-254.

Dezzotti A, R Sbrancia, M Rodríguez-Arias, D Roat, A Parisi. 2003. Regeneración de un bosque mixto de Nothofagus (Nothofagaceae) después de una corta selectiva. Rev. Chilena Historia Natural 76(4): 591-602.

Günes T. 2000. Preoxidase and IAA oxidase activities during rooting in cuttings of three poplar species. Turk $J$ Bot 24: 97-101.

Latsague M, J Lara. 2003. Fenoles solubles totales y su relación con la inhibición de la rizogénesis en estacas de Nothofagus pumilio (Poepp. et Endl.) Krasser. Gayana Botánica 60(2): 90-93.

Latsague M, P Sáez. 2006. Fraccionamiento y recuperación de proteínas solubles libre de fenoles en estacas de lenga (Nothofagus pumilio). Bosque 27(3): 263-266.

Liu Z, H Liu H, H Wang. 1996. Effect of light on endogenous indole -3 - acetic acid, peroxidase and indole -3 - acetic acid oxidase in soybean hypocotyls. Botanical Bulletin of Academia Sinica 37: 113-119.

Mato M, A Vieitez. 1986. Changes in auxin protectors an IAA oxidases during the rooting of chestnut shoots in vitro. Physiol. Plant. 66: 491-494.

Qaddoury A, M Amssa. 2003. Effect of exogenous indole butyric acid on root formation and peroxidase and indole - 3 - acetic acid oxidase activities and phenols contents in date Palm offshoots. Botanical Bulletin of Academia Sinica: 45: 26-31.

Santelices R. 1993. Propagación vegetativa de Raulí, Roble y Coihue a partir de estacas. Ciencia e Investigación Forestal 7(1): 37-47.

Santelices C, A Cabello. 2006. Efecto del ácido indolbutírico, del tipo de la cama de arraigamiento, del substrato y del árbol madre en la capacidad de arraigamiento de estacas de Nothofagus glauca (Phil.) Krasser. Rev. Chilena Historia Natural 79(1): 55-64.

Silva J. 1968. Arraigamiento de estacas de Raulí (Nothofagus obliqua (Poepp. et Endl.) Oerst). Tesis Ingeniero Forestal. Universidad de Chile, Facultad de Agronomía, Escuela de Ingeniería Forestal, Santiago, Chile. 23 p.

Veblen TT, C Donoso. 1987. Alteración y dinámica regenerativa de las especies chilenas de Nothofagus de la Región de Los Lagos. Bosque 8(2): 133-142. 